Preprint typeset in JHEP style - HYPER VERSION

DFPD 02/TH 19

\title{
Wilson line correlators in two-dimensional noncommutative Yang-Mills theory
}

\author{
A. Bassetto* and F. Vian \\ Dipartimento di Fisica "G.Galilei", Via Marzolo 8, 35131 Padova, Italy \\ INFN, Sezione di Padova, Italy \\ E-mail: bassetto@pd.infn.it, vian@pd.infn.it
}

\begin{abstract}
We study the correlator of two parallel Wilson lines in two-dimensional noncommutative Yang-Mills theory, following two different approaches. We first consider a perturbative expansion in the large- $N$ limit and resum all planar diagrams. The second approach is non-perturbative: we exploit the Morita equivalence, mapping the two open lines on the noncommutative torus (which eventually gets decompacted) in two closed Wilson loops winding around the dual commutative torus. Planarity allows us to single out a suitable region of the variables involved, where a saddle-point approximation of the general Morita expression for the correlator can be performed. In this region the correlator nicely compares with the perturbative result, exhibiting an exponential increase with respect to the momentum $p$.
\end{abstract}

Keywords: Noncommutative gauge theories, Wilson lines, large- $N$ limit,

*Partially supported by the European Community network HPRN-CT-2000-00149. 


\section{Introduction}

Correlation functions of gauge invariant local operators in commutative theories provide a lot of information about the dynamics; in the presence of a noncommutative geometry they are even more interesting in view of the intimate merging of space-time and "internal" symmetries [1, 2].

The simplest way of turning ordinary theories into noncommutative ones is to replace the usual multiplication of fields in the Lagrangian with the Moyal $\star$-product. This product is realized by means of a real antisymmetric matrix $\theta^{\mu \nu}$ which parameterizes noncommutativity of Minkowski space-time:

$$
\left[x^{\mu}, x^{\nu}\right]=i \theta^{\mu \nu} \quad \mu, \nu=0, . ., D-1 .
$$

The $\star$-product of two fields $\phi_{1}(x)$ and $\phi_{2}(x)$ is defined as

$$
\phi_{1}(x) \star \phi_{2}(x)=\left.\exp \left[\frac{i}{2} \theta^{\mu \nu} \frac{\partial}{\partial x_{1}^{\mu}} \frac{\partial}{\partial x_{2}^{\nu}}\right] \phi_{1}\left(x_{1}\right) \phi_{2}\left(x_{2}\right)\right|_{x_{1}=x_{2}=x}
$$

and leads to terms in the action with an infinite number of derivatives of fields which make the theory intrinsically non-local. As a consequence, gauge invariance in this case is obtained only after integration over space-time variables and the possibility of having local probes is lost.

It is therefore remarkable that the authors in [3]-[5] have succeeded in proposing a recipe in noncommutative gauge theories which turns local operators into gauge invariant observables carrying a non-vanishing momentum. They showed that an open Wilson line with momentum $p_{\mu}$ is gauge invariant provided the length of the line $l^{\nu}$ is related to the momentum as follows

$$
l^{\nu}=p_{\mu} \theta^{\mu \nu}
$$

Averaging any usual local gauge invariant operator with respect to space-time and group variables with a weight given by an open Wilson line provides an (over-complete) set of dynamical observables in the noncommutative case.

In [5] a perturbative calculation of open Wilson line correlators for a $\mathcal{N}=4$ supersymmetric theory in four dimensions was carried out and compared with dual supergravity results. Ladder diagrams dominate and a good agreement was reached.

In two dimensions the situation is quite different: noncommutativity involves the time variable, but the Lorentz symmetry is not violated owing to the tensorial character of $\theta^{\mu \nu}$. If we choose the light-cone gauge, the perturbative calculation is greatly simplified, thanks to the decoupling of Faddeev-Popov ghosts and to the vanishing of the vector vertices. It turns out that in all diagrams contributing to the line correlators, which are planar according to the 't Hooft's large- $N$ limit [6], $\theta$-dependent phases resulting from non commutativity play no role. 
On the other hand, in two dimensions a remarkable symmetry, the Morita equivalence, allows the mapping of open Wilson lines on a noncommutative torus to closed Wilson loops winding on a dual commutative torus [7]. In a commutative setting Wilson loop correlations can be obtained by geometrical techniques [8]; this opens the possibility of confronting perturbative calculations with non-perturbative solutions, provided a common kinematical region of validity is found for both approaches.

In [9] three basic different regimes have been presented for a noncommutative theory in two dimensions, when approximated by means of a $M \times M$ matrix model. Three different phases (disordered, planar and GMS [10]) are found according to the behaviour of the noncommutativity parameter $\theta$ with respect to the integer $M$ which is to be sent eventually to $\infty\left(\theta \sim M^{\nu}\right.$ with $\nu<1, \nu=1, \nu>1$, respectively). This integer is in turn related to a large distance cutoff of the theory. Owing to the merging of space-time and "internal" symmetries in a noncommutative setting [1, 2], a large- $N$ limit forcefully entails large $M$ and the regimes where a perturbative calculation is likely to make sense are those in which $\theta$ is not going to increase faster than $N$ (the disordered and the planar ones).

Such a perturbative calculation for the correlator of two open Wilson lines is carried out in Sect. 3 in the large- $N$ limit. An exponential increase with the line momentum is found, in agreement with an analogous result in four dimensions [5]. In Sect. 4 the Morita equivalence is exploited together with the geometrical solutions for the partition function and for the loop correlator on the commutative torus. In Sect. 5 the non-perturbative result is considered in the relevant large- $N$ region where a saddle-point approximation provides us with a fairly simple concrete expression for the correlator. In this region, an exponential increase in the momentum is found in agreement with the perturbative result. Outside this region, the saddle-point approximation breaks up, which could be a signal of a transition to a new phase, totally invisible in the perturbative approach. Final comments and remarks are presented in the Conclusions.

\section{Observables in noncommutative gauge theories}

To start with, we set the notation we will use throughout the paper. We will consider the $U(N)$ Yang-Mills theory on a noncommutative two-dimensional space, with classical action

$$
S=-\frac{1}{2} \int d^{2} x \operatorname{Tr} F_{\mu \nu} \star F^{\mu \nu}
$$

where the field strength $F_{\mu \nu}$ is given by

$$
F_{\mu \nu}=\partial_{\mu} A_{\nu}-\partial_{\nu} A_{\mu}-i g\left(A_{\mu} \star A_{\nu}-A_{\nu} \star A_{\mu}\right)
$$

and $A_{\mu}=A_{\mu}^{A} t^{A}$ is a $N \times N$ matrix, normalized as follows: $\operatorname{Tr} t^{A} t^{B}=\frac{1}{2} \delta^{A B}$, capital letters denoting $U(N)$ indices. The $\star$-product was defined in Eq. (1.2). The action Eq. (2.1) is 
invariant under $U(N)$ noncommutative gauge transformations

$$
\delta_{\lambda} A_{\mu}=\partial_{\mu} \lambda-i g\left(A_{\mu} \star \lambda-\lambda \star A_{\mu}\right)
$$

As noticed in [5], under the transformation Eq. (2.3) the operator $\operatorname{Tr} F^{2}(x)$ is not left invariant

$$
\operatorname{Tr} F^{2}(x) \longrightarrow \operatorname{Tr} U(x) \star F^{2}(x) \star U^{\dagger}(x),
$$

with $U(x)=\exp (i g \lambda(x))$. To recover a gauge invariant operator, one has to integrate over all space, since integrals of $\star$-product can be cyclically permuted. In the same fashion a noncommutative Wilson line can be defined by means of the Moyal product as [1], 司]

$$
\Omega_{\star}[x, C]=P_{\star} \exp \left(i g \int_{0}^{l} A_{\mu}(x+\zeta(s)) d \zeta^{\mu}(s)\right)
$$

where $C$ is the curve parameterized by $\zeta(s)$, with $0 \leq s \leq 1, \zeta(0)=0$ and $\zeta(1)=l$, and $P_{\star}$ denotes noncommutative path ordering along $\zeta(s)$ from right to left with respect to increasing $s$ of $\star$-products of functions. The Wilson line is not invariant under a gauge transformation

$$
\Omega_{\star}[x, C] \longrightarrow U(x) \star \Omega_{\star}[x, C] \star U^{\dagger}(x+l),
$$

and moreover cannot be made invariant by closing the line. In fact, recalling that gauge invariance requires integration over coordinates, the following operator

$$
W(p, C)=\int d^{2} x \operatorname{Tr} \Omega_{\star}[x, C] \star e^{i p x},
$$

turns out to be invariant provided $C$ satisfies the condition

$$
l^{\nu}=p_{\mu} \theta^{\mu \nu}
$$

(the Wilson line extends in the direction transverse to the momentum). For simplicity in the following only straight lines will be considered.

It is also easy to see that any local operator $\mathcal{O}(x)$ in ordinary gauge theories admits a noncommutative generalization

$$
\tilde{\mathcal{O}}(p)=\operatorname{Tr} \int d^{2} x \mathcal{O}(x) \star P_{\star} e^{i g \int_{0}^{l} d \zeta A(x+\zeta)} \star e^{i p x}
$$

each of the $\tilde{\mathcal{O}}(p)$ 's being a genuinely different operator at different momentum. Remarkably, at large values of $|p|$, noncommutativity imposes that the length of the Wilson line becomes large and the operator is dominated by the line it is attached to. The correlation function of these operators is thus expected to exhibit an universal large- $p$ behaviour. 


\section{Correlation function of gauge invariant observables}

An interesting quantity to study is the two-point function $\left\langle W(p, C) W^{\dagger}\left(p, C^{\prime}\right)\right\rangle$, where $W(p, C)$ has been defined via Eqs. (2.5), (2.7). It represents the correlation function of two straight parallel Wilson lines of equal length, each carrying a transverse momentum $p$. In four dimensions such a correlator was investigated by Gross et al. [5]. They compared the noncommutative two-point function of Wilson lines (for large $|p|$ and in the 't Hooft limit $N \rightarrow \infty, g^{2} N$ fixed) with the Wilson loop in ordinary gauge theories in the Coulomb phase, which is a function of $T / L$ when the loop is a rectangle of size $T \times L$. There, even though the expansion parameter gets big for large enough $T$, an exponential form is obtained using perturbation theory by summing over ladder diagrams which dominate when $T \gg L$. In four-dimensional noncommutative gauge theories the length of the line $|p \theta|$ plays the role of $T$ and the momentum $|p|$ plays the role of $1 / L$, so that computing the two-point function of the open Wilson lines at large momentum corresponds to computing the expectation value of a rectangular Wilson loop in the commutative case. By resumming the ladder diagrams, the correlator was found to grow exponentially at large momentum, corrections to the leading result having to be ascribed to finite $|p|$ and to subleading terms in $N$. Actually, in four dimensions, at large momentum, the line correlator was shown to provide the universal behaviour of the two-point function of any noncommutative gauge invariant operator.

In two dimensions a similar situation occurs for planar diagrams, where the dependence on $\theta$ intervenes just through the length of the line $l$.

We quantize the theory Eq. (2.1) in the light-cone gauge $A_{-}=0$ at equal times, the free propagator having the following causal expression (WML prescription)

$$
D_{++}^{W M L}(x)=\frac{1}{2 \pi} \frac{x^{-}}{-x^{+}+i \epsilon x^{-}},
$$

first proposed by T.T. Wu [11]. In turn this propagator is nothing but the restriction in two dimensions of the expression proposed by S. Mandelstam and G. Leibbrandt 12 in four dimensions ${ }^{1}$ and derived by means of a canonical quantization in [14].

This form of the propagator allows a smooth transition to an Euclidean formulation, where momentum integrals are performed by means of a "symmetric integration" [11]. Were the theory quantized on the light-front, the following free propagator would ensue

$$
D_{++}^{l-f}(x)=-\frac{i}{2}\left|x^{-}\right| \delta\left(x^{+}\right),
$$

which cannot be smoothly continued to Euclidean variables. Moreover, when combined with the Moyal «-product, it gives rise to singularities which cannot be cured [15]. Therefore the light-front formulation will not be further considered in the sequel.

\footnotetext{
${ }^{1}$ In dimensions higher than two, where physical degrees of freedom are switched on (transverse "gluons"), this causal prescription is the only acceptable one 13$]$.
} 
The light-cone gauge gives rise to other important features like the decoupling of Faddeev-Popov ghosts, which occurs also in the noncommutative case [16], and the absence of the triple gluon vertex in two dimensions. Consequently the computation of the Wilson line correlator is enormously simplified.

With a forethought to the next section, with no loss of generality thanks to the persisting boost invariance, we choose the path $C$ stretching along $x^{0}$, so that $p$ points in the $x^{1}$ direction ${ }^{2}$. In turn the parallel path $C^{\prime}$ is chosen at a distance $\Delta$ from $C$. We begin by expanding the line operators

$$
\begin{aligned}
& W(p, C)=\sum_{j=0}^{\infty}(i g)^{j} \int d^{2} x \int_{\zeta_{j}>\zeta_{j-1}>\ldots>\zeta_{1}}[d \zeta] \operatorname{Tr} A\left(x+\zeta_{1}\right) \star \ldots \star A\left(x+\zeta_{j}\right) \star e^{i p x} \\
& W^{\dagger}\left(p, C^{\prime}\right)=\sum_{j=0}^{\infty}(-i g)^{j} \int d^{2} x \int_{\zeta_{j}^{\prime}>\zeta_{j-1}^{\prime}>\ldots>\zeta_{1}^{\prime}}\left[d \zeta^{\prime}\right] \operatorname{Tr} A\left(x+\zeta_{j}^{\prime}\right) \star \ldots \star A\left(x+\zeta_{1}^{\prime}\right) \star e^{-i p x} .
\end{aligned}
$$

The variables $\zeta$ and $\zeta^{\prime}$ can be conveniently parameterized as $\zeta=(\sigma,-\Delta)$ and $\zeta^{\prime}=\left(\sigma^{\prime}, 0\right)$, with $\sigma=s l^{0}, 0 \leq s \leq 1, \Delta$ a constant quantity in the $x^{1}$-direction and $l^{0}=-\theta p_{1}$, according to Eq. (1.3). Eq. (3.3) can therefore be conveniently rewritten as

$$
\begin{aligned}
W(p, C) & =e^{i p \cdot \Delta} \sum_{j=0}^{\infty}(i g)^{j} \int d^{2} x \int_{\sigma_{j}>\sigma_{j-1}>\ldots>\sigma_{1}}[d \sigma] \operatorname{Tr} A\left(x+\sigma_{1}\right) \star \ldots \star A\left(x+\sigma_{j}\right) \star e^{i p x} \\
W^{\dagger}\left(p, C^{\prime}\right) & =\sum_{j=0}^{\infty}(-i g)^{j} \int d^{2} x \int_{\sigma_{j}^{\prime}>\sigma_{j-1}^{\prime}>\ldots>\sigma_{1}^{\prime}}\left[d \sigma^{\prime}\right] \operatorname{Tr} A\left(x+\sigma_{j}^{\prime}\right) \star \ldots \star A\left(x+\sigma_{1}^{\prime}\right) \star e^{-i p x} .
\end{aligned}
$$

In the following the trivial phase factor $e^{i p \cdot \Delta}$ will be omitted and the dependence on $C$, $C^{\prime}$ will be understood. We then contract the $A$ 's in such a way that the resulting diagram is of leading order in $N$, which yields, according to Eq. (3.1), at a fixed perturbative order $\left(g^{2}\right)^{n}$

$$
(-1)^{n-k}\left(\frac{N}{4 \pi}\right)^{n} \int[d \sigma]\left[d \sigma^{\prime}\right] \int d^{2} x e^{i p x} \prod_{j=1}^{k} \frac{x^{0}+f_{j}\left(\sigma, \sigma^{\prime}, \theta p\right)-x^{1}}{-x^{0}-f_{j}\left(\sigma, \sigma^{\prime}, \theta p\right)-x^{1}}, \quad n \geq 1,
$$

where $k$ is the number of propagators connecting the two lines ${ }^{3}$ and $f_{j}\left(\sigma, \sigma^{\prime}, \theta p\right)$ is a linear function of its variables depending on the topology; the integration region for the $2 n$ geometric variables is understood and the phase factors containing the noncommutativity

\footnotetext{
${ }^{2} \mathrm{~A}$ generic choice is discussed in Appendix B.

${ }^{3}$ The contribution of propagators starting and ending on the same line factorizes and amounts to $(-N /(4 \pi))^{n-k}$.
} 
parameter have been absorbed in the function $f_{j}\left(\sigma, \sigma^{\prime}, \theta p\right)$. We stress that, remarkably, factorization of propagators in coordinate variables occurs just in those diagrams which are dominant at large $N$. This feature in turn makes $\theta$-dependence trivial, as it will be shortly cleared, since it intervenes just through the length of the line $l$. A non-trivial example of a diagram which is leading in $N$ and therefore "planar" in our terminology at $\mathcal{O}\left(g^{6}\right)$ is reported in Appendix A. Diagrams without any propagator connecting the two lines will be disregarded. They provide indeed a contribution concentrated at $p=0$, which is uninteresting for our purposes.

The procedure to be employed to select leading contributions in $N$ will be soon cleared out, but we now concentrate on the volume integration in Eq. (3.5). Surprisingly enough, it will turn out that all integrals will give the same result, no matter what the function $f_{j}\left(\sigma, \sigma^{\prime}, \theta p\right)$ is, i.e. no matter what topology we choose in the set of planar diagrams. This finding is a direct consequence of the integration over the world volume $d^{2} x$, required by noncommutative gauge invariance, and of the orthogonality of the momentum with respect to the direction of the open lines. In so doing the $\theta$-dependence is washed out apart from its occurrence in $l^{0}$.

In order to provide a correct formulation of the theory, continuation to Euclidean variables is required: $x^{0} \rightarrow i x_{2}$; we recall that, to keep the basic algebra unchanged, the noncommutativity parameter $\theta$ has also to be simultaneously continued to an imaginary value: $\theta \rightarrow i \theta$.

A symmetric integration [11, 17] then provides the natural regularization in Eq. (3.5)

$$
\int d^{2} x e^{-i p \cdot x} \prod_{j=1}^{k} \frac{x_{1}+i\left(f_{j}\left(\sigma, \sigma^{\prime}, \theta p\right)+x_{2}\right)}{x_{1}-i\left(f_{j}\left(\sigma, \sigma^{\prime}, \theta p\right)+x_{2}\right)}=(-1)^{k} \frac{4 \pi k}{p^{2}} .
$$

Hence the integration over the geometrical variables in Eq. (3.5) is straightforwardly carried out and yields

$$
\frac{4 \pi k}{p^{2}}\left(\frac{N l^{2}}{4 \pi}\right)^{n} \frac{1}{n_{1} ! n_{2} !},
$$

where $l=|p \theta|$ is the total length of the line and $n_{1}, n_{2}$ are the number of legs stretching out of the first and the second line, respectively $\left(n_{1}+n_{2}=2 n\right)$. (A detailed derivation of Eqs. (3.6) and (3.7) is contained in Appendix B.) As announced, Eq. (3.7) displays a trivial dependence on the topology of the graph, the only remnant being $n_{1} ! n_{2}$ ! in the denominator. Thus, although resumming even only leading contributions in $N$ may have seemed a formidable task when we started, it has now become feasible, provided the exact number of different configurations with fixed $n_{1}, n_{2}$ is known.

One can see from Eq. (3.3) that in the correlator $\left\langle W(p) W^{\dagger}(p)\right\rangle$ the colour factors enter as $\operatorname{Tr}\left[t^{A_{1}} t^{A_{2}} \ldots t^{A_{n_{1}}}\right] \operatorname{Tr}\left[t^{B_{1}} t^{B_{2}} \ldots t^{B_{n_{2}}}\right]$, where $A^{j}, B^{m}$ are indices of $U(N)$ and have to be contracted in pairs so that the result of the product of traces is $(N / 2)^{n}$. Let $j=1, \ldots, 2 q$ be the indices to be contracted inside the first trace and $S_{q}$ the number of ways you can 
form $q$ "good" pairs in it. Imagine you have rearranged the order of matrices in that trace, so that the ones to be coupled are adjacent and come in the first $2 q$ positions. To avoid crossovers, which would reduce the power of $N$, the first element can be paired only with $t^{2 p}, p=1, \ldots, q$. In between these two we are left with $2 p-2$ matrices, and after $t^{2 p}$ with $2 q-2 p$. Therefore we are to face the same problem with a reduced number of elements, namely we have to solve for $S_{p-1}$ and $S_{q-p}$. As a consequence, a recursive relation can be established

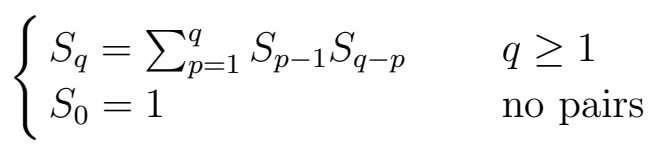

which is solved by

$$
S_{q}=\frac{2^{2 q}}{(q+1) !} \frac{\Gamma\left(q+\frac{1}{2}\right)}{\Gamma\left(\frac{1}{2}\right)}=\left\{\begin{array}{l}
2^{q} \frac{(2 q-1) ! !}{(q+1) !} \\
S_{0}=1 .
\end{array} \quad q \geq 1\right.
$$

Analogously, the number of ways in which we can form $r$ pairs on the second line and maintain the leading power in $N$ is given by $S_{r}$. (Obviously, the number of spare legs on each line has to match, i.e. $r=\frac{n_{2}-n_{1}}{2}+q$.)

If $q, r \neq 0$, the product of traces reduces to

$$
\left(\frac{N}{2}\right)^{q+r} \operatorname{Tr}\left[t^{A_{1}} t^{A_{2}} \ldots t^{A_{k}}\right] \operatorname{Tr}\left[t^{B_{1}} t^{B_{2}} \ldots t^{B_{k}}\right]
$$

where $k=n_{1}-2 q=n_{2}-2 r$. In addition matrices in one of the trace can be cycled (but not swapped!), leading to a factor $k$ in the counting. Eventually, we have to take into account the number of possibilities we had originally to choose a group of $k$ contiguous matrices both from the first and the second trace, which amounts to $n_{1} \cdot n_{2}$. At this stage the resummed contribution to the correlator of graphs with pairs on both lines $\left\langle W(p) W^{\dagger}(p)\right\rangle_{\mathcal{P} \mathcal{P}}$ can be readily written

$$
\begin{aligned}
& \left\langle W(p) W^{\dagger}(p)\right\rangle_{\mathcal{P P}}=\frac{4 \pi}{p^{2}} \sum_{n=1} \sum_{q=1}^{\left[\frac{n_{1}-1}{2}\right]} \sum_{r=1}^{\left[\frac{n_{2}-1}{2}\right]}(-1)^{q+r} \tau^{2 n} k^{2} \cdot n_{1} \cdot n_{2} \cdot S_{q} \cdot S_{r} \cdot \frac{1}{n_{1} !} \cdot \frac{1}{n_{2} !} \\
& =\frac{4 \pi}{p^{2}} \sum_{r=1} \sum_{q=1} \sum_{k=1}(2 i \tau)^{2 q+2 r} \tau^{2 k} \frac{k^{2}}{(q+1) !(r+1) !} \frac{\Gamma\left(q+\frac{1}{2}\right)}{(2 q+k-1) ! \Gamma\left(\frac{1}{2}\right)} \frac{\Gamma\left(r+\frac{1}{2}\right)}{(2 r+k-1) ! \Gamma\left(\frac{1}{2}\right)}
\end{aligned}
$$

where $\tau=\sqrt{\frac{g^{2} N l^{2}}{4 \pi}}$.

Consider then the case with $q=0$ and $r \neq 0$ (or viceversa). Following the instructions provided in the previous example, it is easy to build up the contribution $\left\langle W(p) W^{\dagger}(p)\right\rangle_{\mathcal{P}}$ of graphs with pairs only on one line (recall $k=n_{1}$ )

$$
\left\langle W(p) W^{\dagger}(p)\right\rangle_{\mathcal{P}}=\frac{8 \pi}{p^{2}} \sum_{n=1} \sum_{r=1}^{\left[\frac{n_{2}-1}{2}\right]}(-1)^{r} \tau^{2 n} k \cdot n_{1} \cdot n_{2} \cdot S_{0} \cdot S_{r} \cdot \frac{1}{n_{1} !} \cdot \frac{1}{n_{2} !}=
$$




$$
=\frac{8 \pi}{p^{2}} \sum_{r=1} \sum_{k=1}(-1)^{r} 2^{2 r}\left(\tau^{2}\right)^{r+k} \frac{k}{(r+1) !} \frac{\Gamma\left(r+\frac{1}{2}\right)}{(2 r+k-1) ! \Gamma\left(\frac{1}{2}\right)} \frac{1}{(k-1) !} .
$$

The last piece entering the calculation of $\left\langle W(p) W^{\dagger}(p)\right\rangle$ is due to graphs with no pairs at all $\left(q=r=0\right.$, i.e. $\left.n_{1}=n_{2}=n\right)$, which simply reads

$$
\left\langle W(p) W^{\dagger}(p)\right\rangle_{\mathcal{L}}=\frac{4 \pi}{p^{2}} \sum_{n=1}\left(\tau^{2}\right)^{n} \frac{1}{[(n-1) !]^{2}}=\frac{4 \pi \tau^{2}}{p^{2}} I_{0}(2 \tau) .
$$

Notice that these graphs are ladder graphs in the sense of [5], and only their multiplicity differs from the four-dimensional case. In fact we saw here that a factor $n$ arises due to the cyclicity property of traces. Thereafter, it is not a fortuitous coincidence that our result Eq. (3.13) qualitatively agrees with Eq. (3.24) of Gross et al.

Summing Eqs. (3.11), (3.12), (3.13), after suitable rearrangements, we get

$$
\begin{aligned}
\left\langle W(p) W^{\dagger}(p)\right\rangle=\frac{4 \pi \tau^{2}}{p^{2}} & \left\{2 \sum_{r=1} \frac{(-4)^{r}}{(r+1) !} \frac{\Gamma\left(r+\frac{1}{2}\right)}{\Gamma\left(\frac{1}{2}\right)}\left[\tau I_{2 r+1}(2 \tau)+I_{2 r}(2 \tau)\right]+\right. \\
+ & \left.I_{0}(2 \tau)+\sum_{r=1} r^{2} f_{r}^{2}(\tau)\right\},
\end{aligned}
$$

with $I_{q}(2 \tau)$ a modified Bessel function. The function $f_{r}(\tau)$ can be written as

$$
f_{r}(\tau)=-\frac{1}{4 \pi i} \int_{\gamma-i \infty}^{\gamma+i \infty} d z e^{z \tau} z^{2-r}\left[1+\frac{2}{z^{2}}-\sqrt{1+\frac{4}{z^{2}}}\right], \quad \gamma>0 .
$$

Using an analogous Laplace representation for the Bessel functions, all the sums in Eq. (3.14) can be performed, leading to

$$
\begin{aligned}
& \left\langle W(p) W^{\dagger}(p)\right\rangle=\frac{4 \pi \tau^{2}}{p^{2}}\left[I_{0}(2 \tau)+\int_{\gamma-i \infty}^{\gamma+i \infty} \frac{d z}{8 \pi i} \frac{z+\sqrt{z^{2}-4}}{\sqrt{z^{2}-4}} e^{z \tau}\right. \\
& \times\left(\sqrt{1+\left(z-\sqrt{z^{2}-4}\right)^{2}}-1-\frac{1}{2}\left(z-\sqrt{z^{2}-4}\right)^{2}\right)\left(z+\sqrt{z^{2}-4}+2 \tau\right) \\
& +\int_{\nu_{1}-i \infty}^{\nu_{1}+i \infty} \int_{\nu_{2}-i \infty}^{\nu_{2}+i \infty} \frac{d z d w}{(2 \pi i)^{2}} e^{(z+w) \tau} \frac{z^{3} w^{3}(1+z w)}{4(z w-1)^{3}}\left(1+\frac{2}{z^{2}}-\sqrt{1+\frac{4}{z^{2}}}\right) \\
& \left.\times\left(1+\frac{2}{w^{2}}-\sqrt{1+\frac{4}{w^{2}}}\right)\right],
\end{aligned}
$$

where $\nu_{1}, \nu_{2}>1$ and $\gamma>2$.

One can realize that, at large $\tau$, the term with the double integration dominates and $\left\langle W(p) W^{\dagger}(p)\right\rangle$ increases like $\exp (2 \tau)=\exp \left(\sqrt{g^{2} N l^{2} / \pi}\right)$, disregarding a (small) power correction. As expected, the correlator depends on the 't Hooft coupling $\sqrt{g^{2} N}$; what is 
remarkable is that its asymptotics is an exponential linearly increasing with the line momentum $|p|$. This is reminiscent of what was found by [5] for its four-dimensional analog. Nevertheless, whereas in four dimensions corrections to the leading result have to be ascribed not only to subleading terms in $N$ but also to finite $|p|$, our result Eq. (3.16) is exact and receives corrections only $\mathcal{O}(1 / N)$.

In the next section we will turn our attention to a nonperturbative derivation of the noncommutative Wilson lines correlator, and see how it compares with Eq. (3.16) at large $|p|$. To this regard, it is worth noticing that although Eq. (3.16) follows from a perturbative analysis, having resummed all orders, it holds also at large $g^{2} N$. This fact will prove very useful in the sequel.

The situation here closely resembles the four dimensional case $(\mathcal{N}=4$ NCSYM $)$ [5], where the exponential growth in the line momentum of $\left\langle W(p) W^{\dagger}(p)\right\rangle$ and its dependence on the 't Hooft coupling $\sqrt{g^{2} N}$ allow to extrapolate from the perturbative regime to the strong coupling regime. Many features of this structure are shared also by the dual supergravity description of NCSYM (AdS/CFT correspondence). The parallelism between two-dimensional YM and $(\mathcal{N}=4 \mathrm{SYM})$ is suggested also by the ordinary (i.e. commutative) case. In fact, it was shown in [18 that perturbative computations of the expectation value of a circular Wilson loop and a pair of infinite anti-parallel lines, obtained by resumming an infinite class of ladder-like planar diagrams, when extrapolated to strong coupling, produce an expectation value peculiar of the results of AdS/CFT correspondence, namely $\langle W\rangle \sim \exp \left(\right.$ const. $\left.\sqrt{g^{2} N}\right)$. Though resummation is a very complicated problem, in general, simplifications occur thanks to supersymmetry. One could be tempted to conjecture that the absence of transverse degrees of freedom and the finiteness of the theory in two dimensions play the role of supersymmetry in dimension four.

\section{An approach based on the Morita equivalence}

When both coordinates are compactified to form a torus, a remarkable symmetry, called Morita equivalence [7], relates different noncommutative gauge theories living on different noncommutative tori: the duality group $S O(2,2, \mathbf{Z})$ has an $S L(2, \mathbf{Z})$ subgroup which acts as follows

$$
\begin{aligned}
\left(\begin{array}{l}
m^{\prime} \\
N^{\prime}
\end{array}\right)=\left(\begin{array}{ll}
a & b \\
c & d
\end{array}\right)\left(\begin{array}{l}
m \\
N
\end{array}\right), \quad \Theta^{\prime}=\frac{c+d \Theta}{a+b \Theta}, \\
\left(R^{\prime}\right)^{2}=R^{2}(a+b \Theta)^{2}, \quad\left(g^{\prime}\right)^{2}=g^{2}|a+b \Theta|, \quad \tilde{\Phi}^{\prime}=(a+b \Theta)^{2} \tilde{\Phi}-b(a+b \Theta),
\end{aligned}
$$

where $\Theta \equiv \theta /\left(2 \pi R^{2}\right), \tilde{\Phi} \equiv 2 \pi R^{2} \Phi, \Phi$ being a background connection and $R$ the radius of the torus, which, for simplicity, we assume to be square. The first entry $m$ denotes the magnetic flux, while $N$ characterizes the gauge group $U(N)$. It is not restrictive to consider the quantities $m$ and $\theta$ to be positive. The parameters of the transformation are integers, constrained by the condition $a d-b c=1$. 
The map in the equations above is flexible enough to allow for a commutative theory on the second torus by choosing $\Theta^{\prime}=0$. As a consequence, the parameter $d$ will be set equal to $-c / \Theta, \Theta$ being a suitable rational quantity. In the sequel for notational convenience all the primed quantities will acquire the subscript $c\left(N^{\prime} \equiv N_{c}, m^{\prime} \equiv m_{c}, \ldots\right)$.

We are eventually interested in a noncommutative theory defined on a plane $(R \rightarrow \infty)$, with a trivial first Chern class $(m=0, \Phi=0)$ and a gauge group $U(N)$ with a large $N$, since we want to establish a comparison with the perturbative approach of the previous section. Eqs. (4.1), (4.2) together with the unimodularity condition allow us to eliminate the parameters $a, b$ and $c$ in terms of "physical" quantities, namely $a=\frac{N}{N_{c}}-\frac{\Theta m_{c}}{N}, b=\frac{m_{c}}{N}$ and $c=-\frac{\Theta N_{c}}{N}$. The quantity $d=\frac{N_{c}}{N}$ is a positive integer; as a consequence $N_{c}$ will also assume large values. Moreover, it follows that $m_{c}$ will be an integer multiple of $N, \Theta d$ will be integer and $\Theta d \frac{m_{c}}{N}-1$ will be an integer multiple of $d$. All these constraints are compatible with suitable rational values of $\Theta$.

Finally, the following relations between the radii of the tori and between the coupling constants

$$
R_{c}=\frac{R}{d}, \quad g_{c}^{2}=\frac{g^{2}}{d}
$$

will ensue. It is also useful to introduce the dimensionless quantity

$$
\mathcal{A}=4 \pi^{2}\left(g_{c} R_{c}\right)^{2}=4 \pi^{2} \frac{(g R)^{2}}{d^{3}}
$$

related to the total area of the commutative torus.

On the commutative torus we can develop the $U\left(N_{c}\right)$ Yang-Mills theory, in particular compute its partition function, according to a well established geometrical procedure [8].

We start by considering the action

$$
S=\frac{1}{4 g_{c}^{2}} \int d^{2} x \operatorname{Tr}\left[\left(F_{\mu \nu}-\frac{m_{c}}{2 \pi R_{c}^{2} N_{c}} \epsilon_{\mu \nu} \mathbf{I}\right)\left(F^{\mu \nu}-\frac{m_{c}}{2 \pi R_{c}^{2} N_{c}} \epsilon^{\mu \nu} \mathbf{I}\right)\right],
$$

where the explicit expression for the background connection $\Phi_{c}=-\frac{m_{c}}{2 \pi R_{c}^{2} N_{c}} \mathbf{I}$ has been introduced.

The quantity $m_{c}$ is just the first Chern class of the $U\left(N_{c}\right)$ field

$$
m_{c}=\frac{1}{4 \pi} \int d^{2} x \operatorname{Tr}\left[F_{\mu \nu} \epsilon^{\mu \nu}\right]
$$

the action thereby becomes

$$
S=\frac{1}{4 g_{c}^{2}} \int d^{2} x \operatorname{Tr}\left[F_{\mu \nu} F^{\mu \nu}\right]-\frac{2 \pi^{2} m_{c}^{2}}{\mathcal{A} N_{c}}
$$

The general Migdal's formula for the partition function on a torus reads [8

$$
\mathcal{Z}=\sum_{\mathcal{R}} \exp \left[-\frac{\mathcal{A}}{2} C_{2}(\mathcal{R})\right]
$$


$C_{2}$ being the second Casimir operator in the representation $\mathcal{R}$. The sum runs over all the irreducible representations of the gauge group $U\left(N_{c}\right)$, which can be labelled by a strongly decreasing sequence of integers $n_{1}>n_{2}>\ldots>n_{N_{c}}$, related to a Young tableau. In terms of such integers, the Casimir operator reads

$$
C_{2}(\mathcal{R})=\sum_{i=1}^{N_{c}}\left(n_{i}-\frac{N_{c}-1}{2}\right)^{2}-\frac{N_{c}\left(N_{c}^{2}-1\right)}{12}
$$

The constant term only affects the overall normalization in our case and, therefore, will be disregarded in the sequel. By exploiting the invariance under permutations, Eq. (4.5) can be written as

$$
\mathcal{Z}=\frac{1}{N_{c} !} \sum_{n_{1} \neq n_{2} \neq \ldots n_{N_{c}}} \exp \left[-\frac{\mathcal{A}}{2} \sum_{i=1}^{N_{c}}\left(n_{i}-\frac{N_{c}-1}{2}\right)^{2}\right]
$$

In order to fix the Chern class $m_{c}$ we have to factorize the partition function according to the decomposition $U\left(N_{c}\right)=U(1) \times S U\left(N_{c}\right) / Z_{N_{c}}$. This can be achieved introducing the integers

$$
\bar{n}_{i}=n_{i}-n_{1}, \quad i=2, \ldots, N_{c}
$$

Eq. (4.7) becomes

$$
\begin{aligned}
\mathcal{Z}=\frac{1}{N_{c} !} \sum_{n_{1}=-\infty}^{+\infty} \sum_{\bar{n}_{i} \neq \bar{n}_{j} \neq 0} \exp & {\left[-\frac{\mathcal{A} N_{c}}{2}\left(n_{1}-\frac{N_{c}-1}{2}+\frac{1}{N_{c}} \sum_{i=2}^{N_{c}} \bar{n}_{i}\right)^{2}\right] \times } \\
\exp & {\left[-\frac{\mathcal{A}}{2}\left(\sum_{i=2}^{N_{c}} \bar{n}_{i}^{2}-\frac{1}{N_{c}}\left(\sum_{i=2}^{N_{c}} \bar{n}_{i}\right)^{2}\right)\right] }
\end{aligned}
$$

Using standard techniques, Eq. (4.8) can be rewritten as

$$
\begin{aligned}
\mathcal{Z} & =\frac{1}{N_{c} N_{c} !} \sum_{l, k=0}^{N_{c}-1} \sum_{n_{1}=-\infty}^{+\infty} \exp \left[-\frac{\mathcal{A} N_{c}}{2}\left(n_{1}+\frac{l}{N_{c}}\right)^{2}\right] \\
& \times \sum_{\bar{n}_{i} \neq \bar{n}_{j} \neq 0} \exp \left[-\frac{\mathcal{A}}{2}\left(\sum_{i=2}^{N_{c}} \bar{n}_{i}^{2}-\frac{1}{N_{c}}\left(\sum_{i=2}^{N_{c}} \bar{n}_{i}\right)^{2}\right)-2 \pi i k\left(\frac{l}{N_{c}}+\frac{N_{c}-1}{2}-\frac{1}{N_{c}} \sum_{i=2}^{N_{c}} \bar{n}_{i}\right)\right] .
\end{aligned}
$$

After a Poisson resummation over $n_{1}$, the partition function becomes

$$
\mathcal{Z}=\sqrt{\frac{2 \pi}{\mathcal{A} N_{c}}} \sum_{k=0}^{N_{c}-1} \sum_{n_{1}=-\infty}^{+\infty} \delta_{N_{c}}\left(n_{1}-k\right) \exp \left[-\frac{2 \pi^{2} n_{1}^{2}}{\mathcal{A} N_{c}}\right] \mathcal{Z}_{k}
$$


$\delta_{N}$ being the $N$-periodic delta-function and

$$
\mathcal{Z}_{k}=\frac{1}{N_{c} !} \sum_{\bar{n}_{i} \neq \bar{n}_{j} \neq 0} \exp \left[-\frac{\mathcal{A}}{2}\left(\sum_{i=2}^{N_{c}} \bar{n}_{i}^{2}-\frac{1}{N_{c}}\left(\sum_{i=2}^{N_{c}} \bar{n}_{i}\right)^{2}\right)-2 \pi i k\left(\frac{N_{c}-1}{2}-\frac{1}{N_{c}} \sum_{i=2}^{N_{c}} \bar{n}_{i}\right)\right],
$$

being the $S U\left(N_{c}\right)$ partition function in the $k$-th 't Hooft sector $[19$.

By retaining only the term with $k=m_{c}$ and cancelling the $U(1)$ contribution against the background connection, after restoring the full permutation symmetry with respect to the integers $n_{i}$, we obtain the final expression

$$
\begin{aligned}
\mathcal{Z} & =\sqrt{\frac{2 \pi}{\mathcal{A} N_{c}}} \frac{1}{N_{c} !} \sum_{n_{i} \neq n_{j}} \exp \left[-\frac{\mathcal{A}}{2}\left(\sum_{i=1}^{N_{c}} n_{i}^{2}-\frac{1}{N_{c}}\left(\sum_{i=1}^{N_{c}} n_{i}\right)^{2}\right)\right] \\
& \times \int_{0}^{2 \pi} \frac{d \alpha}{\sqrt{\pi}} \exp \left[-\left(\alpha-\frac{2 \pi}{N_{c}} \sum_{i=1}^{N_{c}} n_{i}\right)^{2}-2 \pi i m_{c}\left(\frac{N_{c}-1}{2}-\frac{1}{N_{c}} \sum_{i=1}^{N_{c}} n_{i}\right)\right] .
\end{aligned}
$$

Now we turn our attention to the calculation of gauge invariant observable quantities of the noncommutative theory; we are interested here on the correlation function of two straight parallel Wilson lines of equal length, at a distance $\Delta$, lying on the noncommutative torus without winding around it, each carrying a transverse momentum $p$ [20, 21]. The noncommutative torus will eventually be decompacted by sending its radius $R \rightarrow \infty$.

The operator for an open Wilson line on the noncommutative plane has been defined in Eq. (2.7). On the noncommutative torus, with the line $C$ stretching along $x_{2}$, we have the expression

$$
W(k, C)=\frac{1}{4 \pi^{2} R^{2}} \int_{0}^{2 \pi R} d^{2} x \Omega_{\star}[x, C] \star \exp \left(i k x_{1} / R\right),
$$

where $k$ is the integer associated to the transverse momentum $p=\frac{k}{R}$. The no-winding condition entails the constraint $\theta k<2 \pi R^{2}$, namely $l=p \theta<2 \pi R, l$ being the total length of the straight line. The normalization of $W(k)$ is chosen so that $W(0)=1$.

Now we exploit again the Morita equivalence in order to map the open Wilson line on the noncommutative torus on a closed Polyakov loop of the ordinary Yang-Mills theory winding $k$ times around the commutative torus in the $x_{2}$ direction

$$
\begin{aligned}
W(k) & =W^{(k)}, \\
W^{(k)} & =\frac{1}{4 \pi^{2} R_{c}^{2}} \int_{0}^{2 \pi R_{c}} d^{2} x \frac{1}{N_{c}} \operatorname{Tr}\left[\Omega^{(k)}\left(x_{1}\right)\right] .
\end{aligned}
$$

The trace is to be taken in the fundamental representation of $U\left(N_{c}\right)$ and $\Omega^{(k)}\left(x_{1}\right)$ is the holonomy of the closed path [22, 20, 21]. This holonomy is to be computed in the flux 
sector $m_{c}$, singled out in the decomposition $U\left(N_{c}\right)=U(1) \times S U\left(N_{c}\right) / Z_{N_{c}}$. Again the $U(1)$ contribution is taken in the trivial sector and cancels against the background connection in the classical action.

The correlation function of two straight parallel open Wilson lines $C, C^{\prime}$, at a distance $\Delta$ in the $x_{1}$-direction reads [2]

$$
\begin{aligned}
& \mathcal{W}_{2}(k) \equiv<W(k, C) W\left(-k, C^{\prime}\right)> \\
= & \exp (i p \cdot \Delta) \frac{1}{2 \pi R_{c}} \int_{0}^{2 \pi R_{c}} d x<\frac{1}{N_{c}} \operatorname{Tr}\left[\Omega^{(k)}(x)\right] \frac{1}{N_{c}} \operatorname{Tr}\left[\Omega^{(-k)}(0)\right]>,
\end{aligned}
$$

where translational invariance in the $x_{1}$-direction has been taken into account and thereby three trivial integrations have been performed. The normalization in Eq. (4.15) is such that $\mathcal{W}_{2}(0)=1$. Again the trivial phase factor $\exp (i p \cdot \Delta)$ will be disregarded.

The integrand in Eq. (4.15) can be computed by resorting to the Migdal-Rusakov's formula for $U\left(N_{c}\right)$

$$
\begin{aligned}
& \frac{1}{N_{c}^{2}}<\operatorname{Tr}\left[\Omega^{(k)}(x)\right] \operatorname{Tr}\left[\Omega^{(-k)}(0)\right]>=\frac{1}{\mathcal{Z} N_{c}^{2}} \sum_{\mathcal{R S}} \exp \left[-\frac{\mathcal{A}}{2}\left(1-\frac{x}{2 \pi R_{c}}\right) C_{2}(\mathcal{R})\right] \\
& \times \exp \left[-\frac{\mathcal{A}}{2} \frac{x}{2 \pi R_{c}} C_{2}(\mathcal{S})\right] \int d U_{1} \chi_{\mathcal{R}}\left(U_{1}\right) \chi_{F}\left(U_{1}^{k}\right) \chi_{\mathcal{S}}^{\dagger}\left(U_{1}\right) \int d U_{2} \chi_{\mathcal{S}}\left(U_{2}\right) \chi_{F}\left(U_{2}^{-k}\right) \chi_{\mathcal{R}}^{\dagger}\left(U_{2}\right),
\end{aligned}
$$

$\chi_{F}$ being the character of the fundamental representation of $U\left(N_{c}\right)$.

By repeating the procedure we have followed in computing the partition function, keeping again the projection onto the $m_{c}$ sector in the decomposition $U\left(N_{c}\right)=U(1) \times$ $S U\left(N_{c}\right) / Z_{N_{c}}$ and subtracting the classical background, we get

$$
\begin{aligned}
& \frac{1}{N_{c}^{2}}<\operatorname{Tr}\left[\Omega^{(k)}(x)\right] \operatorname{Tr}\left[\Omega^{(-k)}(0)\right]>=\frac{1}{\mathcal{Z} N_{c} !} \sqrt{\frac{2 \pi}{\mathcal{A} N_{c}}} \exp \left[-\frac{k^{2} x \mathcal{A}}{4 \pi R_{c}}\left(1-\frac{x}{2 \pi R_{c} N_{c}}\right)\right] \\
& \times \sum_{n_{i} \neq n_{j}} \exp \left[-\frac{\mathcal{A}}{2}\left(\sum_{i=1}^{N_{c}} n_{i}^{2}-\frac{1}{N_{c}}\left(\sum_{i=1}^{N_{c}} n_{i}\right)^{2}\right)\right] \frac{1}{N_{c}} \sum_{j=1}^{N_{c}} \exp \left[-\frac{x k \mathcal{A}}{2 \pi R_{c}}\left(n_{j}-\frac{1}{N_{c}} \sum_{i=1}^{N_{c}} n_{i}\right)\right] \\
& \times \int_{0}^{2 \pi} \frac{d \alpha}{\sqrt{\pi}} \exp \left[-\left(\alpha-\frac{2 \pi}{N_{c}} \sum_{i=1}^{N_{c}} n_{i}\right)^{2}-2 \pi i m_{c}\left(\frac{N_{c}-1}{2}-\frac{1}{N_{c}} \sum_{i=1}^{N_{c}} n_{i}\right)\right] .
\end{aligned}
$$

\section{The planar phase}

Eqs. (4.12), (4.17) entail $N_{c}$ sums over the different integers $n_{i}$ which can take any value between $-\infty$ and $+\infty$. To this regard they might be interpreted as mimicking a fermionic model, the $n_{i}$ labelling the energetic levels which can be either empty or singly occupied. As a consequence of the $S U\left(N_{c}\right) / Z_{N_{c}}$ symmetry, those equations are manifestly invariant under a simultaneous shift of all the $n_{i}$ by an integer. 
Obviously, plenty of different configurations are possible and to sum over all of them is beyond reach. We are therefore seeking for configurations which may be dominant in particular physical regimes. In a recent paper [9], three basic different regimes have been presented for a scalar noncommutative theory in two dimensions, when approximated by means of a $M \times M$ matrix model. Three different phases (disordered, planar and GMS [10]) are possible according to the behaviour of the noncommutativity parameter $\theta$ with respect to the integer $M$ which is to be sent eventually to $\infty\left(\theta \sim M^{\nu}\right.$ with $\nu<1, \nu=1, \nu>1$, respectively). This integer in turn is related to a large distance cutoff $L$ of the theory, which can be identified with the length of the side of a square torus.

In our case we are facing a gauge theory: we have previously obtained a perturbative expression for the two Wilson line correlator on the noncommutative plane. In order to justify keeping only planar diagrams, we have to resort to a large- $N$ approximation, $U(N)$ being the relevant gauge group.

It is not granted a priori that a comparison between perturbative and non-perturbative results may be possible at all. The common wisdom is that a comparison (if any) may concern the planar phase; this was actually the finding for $\mathcal{N}=4$ NCSYM in four dimensions, via AdS/CFT correspondence [5].

In two dimensions as well dramatic simplifications are expected to take place, thanks to the absence of transverse degrees of freedom and to the onset of the invariance under area-preserving diffeomorphisms. Then the Morita equivalence should provide the required bridge.

On the non-perturbative side some constraints may be imposed; coming back to the quantities introduced in the previous section to parameterize the Morita transformation, we start by considering large $N$ values in order to comply with our perturbative treatment of Sect. 3; this forces even larger values for $N_{c}$, the ratio $d$ being a positive integer, and a large value for the "commutative" flux $m_{c}$.

Eqs. (4.12), 4.17) exhibit a Gaussian damping with respect to the "occupation numbers" $n_{i}$, which suggests that a kind of saddle-point approximation may be feasible. The most favoured configurations are those with minimal fluctuations. This happens when the integers $n_{i}$ assume adjacent values.

Thanks to the invariance under a global translation of the $n_{i}$ 's and choosing for the sake of simplicity an odd value for $N_{c}$, we can set $\sum_{i=1}^{N_{c}} n_{i}=0$; the other equivalent configurations will be disregarded as they will eventually cancel in normalized quantities like correlators. Eq. (4.12) then becomes

$$
\mathcal{Z} \simeq \sqrt{\frac{2 \pi}{\mathcal{A} N_{c}}} \frac{1}{N_{c} !} \exp \left[-\frac{\mathcal{A}}{2} \frac{\left(N_{c}-1\right) N_{c}\left(N_{c}+1\right)}{12}\right] \int_{0}^{2 \pi} \frac{d \alpha}{\sqrt{\pi}} \exp \left(-\alpha^{2}\right) .
$$

Other sets of $n_{i}$ lead to an extra suppression factor behaving at least as $\exp \left(-\mathcal{A} N_{c}\right)$.

Now we turn our attention to the two line correlator, namely to Eq. (4.17). In order to retain the damping character of the overall exponential factor, we must require that 
$|k|$ is not increasing faster than $N_{c}^{2}$. However, the configurations with the $n_{i}$ 's assuming adjacent values remain the most relevant ones for the correlator only provided $|k|$ is not increasing faster than $N_{c}$. As a matter of fact, in Eq. (4.17) different configurations would produce competing exponential corrections behaving as $\exp \left(-\mathcal{A} N_{c} \delta\right)$ and $\exp (|k| \mathcal{A} \delta), \delta$ being a typical gap in the distribution of the $n_{i}$ 's. Then, if $|k|<N_{c}$, Eq. (4.17) can be approximated following the same procedure we applied to derive Eq. (5.1]), leading to

$$
\begin{aligned}
& \frac{1}{N_{c}^{2}}<\operatorname{Tr}\left[\Omega^{(k)}(x)\right] \operatorname{Tr}\left[\Omega^{(-k)}(0)\right]>\simeq \frac{1}{\mathcal{Z} N_{c} !} \sqrt{\frac{2 \pi}{\mathcal{A} N_{c}}} \exp \left[-\frac{k^{2} x \mathcal{A}}{4 \pi R_{c}}\left(1-\frac{x}{2 \pi R_{c} N_{c}}\right)\right] \\
& \times \exp \left[-\frac{\mathcal{A}\left(N_{c}-1\right) N_{c}\left(N_{c}+1\right)}{24}\right] \frac{1}{N_{c}} \frac{\sinh \frac{x|k| \mathcal{A} N_{c}}{4 \pi R_{c}}}{\sinh \frac{x|k| \mathcal{A}}{4 \pi R_{c}}} \int_{0}^{2 \pi} \frac{d \alpha}{\sqrt{\pi}} \exp \left(-\alpha^{2}\right) .
\end{aligned}
$$

Taking Eq. (5.1) into account, we eventually get, for large values of $N_{c}$,

$$
\frac{1}{N_{c}^{2}}<\operatorname{Tr}\left[\Omega^{(k)}(x)\right] \operatorname{Tr}\left[\Omega^{(-k)}(0)\right]>\simeq \frac{1}{N_{c}} \exp \left[\frac{x \mathcal{A}}{4 \pi R_{c}}|k|\left(N_{c}-1-|k|\right)\right] .
$$

Introducing Eq. (5.3) in Eq. (4.15) and performing the integration over $x$, we obtain

$$
\mathcal{W}_{2}(k) \equiv<W(k) W(-k)>\simeq \frac{\exp \left[\frac{\mathcal{A}}{2}|k|\left(N_{c}-1-|k|\right)\right]-1}{\frac{\mathcal{A}}{2} N_{c}|k|\left(N_{c}-1-|k|\right)} .
$$

Remarkably, when $|k|<N_{c}-1$, we find a correlation function exponentially increasing with $|k|$, in qualitative agreement with an analogous finding in [5] and with our perturbative result in Sect. 3

$$
\mathcal{W}_{2}(k) \equiv<W(k) W(-k)>\simeq \frac{\exp \left(\frac{\mathcal{A}}{2}|k| N_{c}\right)}{\frac{\mathcal{A}}{2}|k| N_{c}^{2}} .
$$

In order to reach a quantitative agreement we can consider a fine tuning of the exponents. When comparing Eq. (4.17) in the large- $N$ approximation with Eq. (3.16), one might argue that their normalizations are different; actually Eq. (3.16) could also be normalized to be unity at $p=0$; however this would only entail a trivial power factor.

If we trade the quantities $\mathcal{A}$ and $N_{c}$ for $\theta, R$ and $|c|$, by means of their expressions (see the previous section)

$$
\mathcal{A}=\frac{g^{2} \theta^{3}}{2 \pi R^{4}|c|^{3}}, \quad N_{c}=\frac{1}{\theta} 2 \pi R^{2} N|c|
$$

we get

$$
\mathcal{W}_{2}(k) \simeq \frac{\exp \left(p \frac{g^{2} N \theta^{2}}{2 R|c|^{2}}\right)}{p \frac{g^{2} N \theta^{2}}{2 R|c|^{2}}}
$$

Agreement with the asymptotic behaviour of Eq. (3.16) suggests

$$
\theta \sim \frac{2 R c^{2}}{g \sqrt{N \pi}}
$$


Let us summarize at this point the various requirements to be met for the consistency of our procedure. The radius $R$ of the noncommutative torus has to be sent to $\infty$, since we want to reach eventually the noncommutative plane, as well as $N$ of $U(N)$, so that planarity is entailed. The validity of the saddle-point approximation we have hitherto considered requires

$$
\mathcal{A} N_{c} \sim c^{2} \rightarrow \infty
$$

On the other hand, the no-winding condition $p \theta<2 \pi R$ we have imposed in our nonperturbative approach entails that $\theta$ cannot increase faster than $R$ when we consider nonvanishing values of the transverse momentum. Hence, from Eq. (5.8), it follows

$$
g \sqrt{N} \gtrsim c^{2} p
$$

which implies that, for finite $p$, the 't Hooft coupling should be large in this limit.

In truth, the occurrence of a strong ('t Hooft) coupling regime is not surprising. We already noticed in Sect. 3 how the result Eq. (3.16), though following from a perturbative analysis, holds also at large values of $g^{2} N$. The situation here closely resembles the four dimensional case $(\mathcal{N}=4 \mathrm{NCSYM})$ [5], in the fact that the exponential growth in the line momentum of $\left\langle W(p) W^{\dagger}(p)\right\rangle$ and its dependence on the 't Hooft coupling $\sqrt{g^{2} N}$ allow to extrapolate from the perturbative regime to the strong coupling regime.

If we remember that $R$ is the natural cutoff of our formulation, the condition $\theta \sim R^{\nu}$ with $\nu \leq 1$ is reminiscent of the analogous condition in 9] with respect to the cutoff $M$ (or the torus side length $L$ ), related to the dimension of their matrix model. Actually $\nu<1$ describes the disordered phase, where quantum effects are dominant, while $\nu=1$ is a border-line value, related to the so-called planar phase. The values $\nu>1$ would correspond to the GMS phase which is inaccessible to our treatment.

As a final remark we notice that Eq. (5.4) changes dramatically when $|k|>N_{c}-1$, strongly deviating from the perturbative result and thus possibly suggesting the onset of a new phase. Nevertheless, we ought to recall that in this region the saddle-point approximation we adopted no longer holds.

\section{Conclusions}

Two quite different approaches were pursued to calculate the correlator of two parallel Wilson lines in two space-time dimensions. In the first one we considered a perturbative expansion and were able to resum all planar diagrams, which is justified as far as the large- $N$ limit is concerned. For planar diagrams, $\theta$-dependent phases resulting from noncommutativity play no role. This is indeed a particular phenomenon occurring only in two dimensions, where transverse degrees of freedom are absent and the theory exhibits invariance under area-preserving diffeomorphisms. 
In spite of these peculiarities, we still find a correlation function which increases exponentially with respect to the momentum associated to the lines, in analogy with the result for $\mathcal{N}=4$ NCSYM in four dimensions found in [5] in the planar regime. We conjecture that the simplifying role of supersymmetry is replaced in two dimensions by the triviality of the phase space.

On the non-perturbative side in four dimensions a comparison with supergravity results was possible via AdS/CFT correspondence. In two dimensions we exploited the Morita equivalence in order to map the open lines on the noncommutative torus (which eventually gets decompacted) into two closed Wilson loops winding around the dual commutative torus. In turn, in a commutative setting, the correlator can be obtained by well known geometrical techniques; this provides a non-perturbative solution and open the possibility of a comparison with the perturbative result, provided they share a kinematical region of validity. The common wisdom is that a comparison may be possible when the theory is in the planar phase; this was indeed confirmed for $\mathcal{N}=4$ NCSYM in four dimensions via AdS/CFT correspondence.

In two dimensions planarity allows us to perform a saddle-point approximation on the general Morita expression for the correlator, which nicely compares with the perturbative result, exhibiting an exponential increase with respect to the momentum $p$ in the region $k \lesssim N_{c}$. Beyond such a value, the approximation we used can no longer be trusted, possibly calling for a new regime. To clarify and properly understand this issue looks exciting and promises further interesting insights into the theory.

\section{Acknowledgments}

Discussions with G. De Pol and L. Griguolo are gratefully acknowledged. One of us (F.V.) is pleased to thank K. Zarembo for helpful comments.

\section{Appendix A}

The diagram in Fig.1 entails the following integral in momentum space

$$
\begin{aligned}
I= & \int \frac{d^{2} p_{1}}{\left[p_{1-}\right]^{2}} \frac{d^{2} p_{2}}{\left[p_{2-}\right]^{2}} \frac{d^{2} p_{3}}{\left[p_{3-}\right]^{2}} \delta^{(2)}\left(p_{1}+p_{2}+p_{3}-p\right) \\
& \times \exp \left[-i p_{1}\left(\zeta_{1}-\zeta_{3}^{\prime}\right)-i p_{2}\left(\zeta_{2}-\zeta_{1}^{\prime}\right)-i p_{3}\left(\zeta_{3}-\zeta_{2}^{\prime}\right)+i\left(p_{1} \theta p_{2}+p_{1} \theta p_{3}\right)\right],
\end{aligned}
$$

where $p_{-} \equiv p_{x}-i p_{y}$ in Euclidean components. An easy calculation leads to

$$
\begin{aligned}
I=-\frac{\pi}{4} \int d^{2} x & e^{-i p x} \frac{x_{1}+i\left(x_{2}-\zeta_{2}+\zeta_{1}^{\prime}\right)}{x_{1}-i\left(x_{2}-\zeta_{2}+\zeta_{1}^{\prime}\right)} \\
& \times \frac{x_{1}+i\left(x_{2}-\zeta_{3}+\zeta_{2}^{\prime}\right)}{x_{1}-i\left(x_{2}-\zeta_{3}+\zeta_{2}^{\prime}\right)} \times \frac{x_{1}+i\left(x_{2}-\zeta_{1}+\zeta_{3}^{\prime}-\theta p\right)}{x_{1}-i\left(x_{2}-\zeta_{1}+\zeta_{3}^{\prime}-\theta p\right)},
\end{aligned}
$$




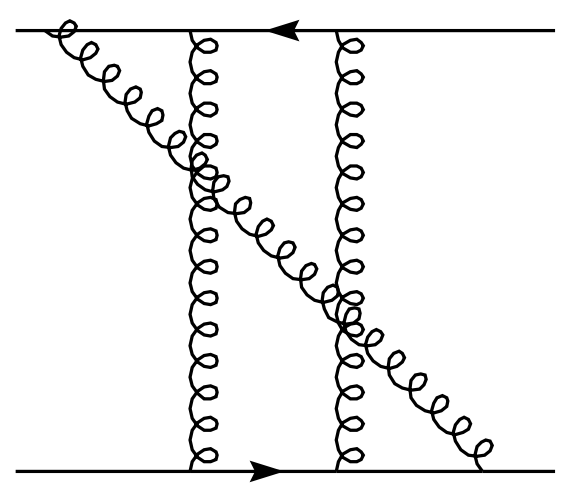

Figure 1: A non-trivial leading diagram in the large- $N$ limit.

which has precisely the form of the left-hand side of Eq. (3.6). Integration over the $\zeta$ 's has to be subsequently performed.

\section{Appendix B}

We start by proving Eq. (3.6). Let us define

$$
I_{k}=\int d^{2} x e^{-i p x} \prod_{j=1}^{k} \frac{x_{1}+F_{1, j}\left(\zeta, \zeta^{\prime}, \theta p\right)+i\left(F_{2, j}\left(\zeta, \zeta^{\prime}, \theta p\right)+x_{2}\right)}{x_{1}+F_{1, j}\left(\zeta, \zeta^{\prime}, \theta p\right)-i\left(F_{2, j}\left(\zeta, \zeta^{\prime}, \theta p\right)+x_{2}\right)},
$$

where $F_{j}\left(\zeta, \zeta^{\prime}, \theta p\right)=F_{1, j}\left(\zeta, \zeta^{\prime}, \theta p\right)+i F_{2, j}\left(\zeta, \zeta^{\prime}, \theta p\right)$ are linear functions of their variables according to the topology of the diagram. This is a more general version of the integral in Eq. (3.6), since we allow the parallel lines, parametrized by $\zeta, \zeta^{\prime}$, to form an arbitrary angle with the frame and thus the functions $F_{j}$ become complex. By means of a recursive argument the product of denominators in Eq. (8.1) can be easily simplified as

$$
\prod_{j=1}^{k} \frac{1}{x_{1}+F_{j}^{*}\left(\zeta, \zeta^{\prime}, \theta p\right)-i x_{2}}=\sum_{m=1}^{k} \prod_{j \neq m} \frac{1}{F_{j}^{*}-F_{m}^{*}} \frac{1}{x_{1}+F_{m}^{*}-i x_{2}} .
$$

Inserting Eq. (8.2) in Eq. (8.1) and perfoming a shift in the integration variables such that $x_{1} \mapsto x_{1}+F_{1, m}, x_{2} \mapsto x_{2}+F_{2, m}$, we get

$$
\begin{aligned}
I_{k} & =\sum_{m=1}^{k} \prod_{j \neq m} \frac{1}{F_{j}^{*}-F_{m}^{*}} \int d^{2} x e^{-i p x} \frac{\prod_{l=1}^{k}\left(x_{1}+F_{l}-F_{m}+i x_{2}\right)}{x_{1}-i x_{2}} \\
& =\sum_{m=1}^{k} \sum_{q=0}^{k-1} \sum_{j_{1}<j_{2}<\ldots<j_{q}}\left(F_{j_{1}}-F_{m}\right)\left(F_{j_{2}}-F_{m}\right) \ldots\left(F_{j_{q}}-F_{m}\right)
\end{aligned}
$$




$$
\times \prod_{j \neq m} \frac{1}{F_{j}^{*}-F_{m}^{*}} \int d^{2} x e^{-i p x} \frac{\left(x_{1}+i x_{2}\right)^{k-q}}{x_{1}-i x_{2}},
$$

where the sum is over all possible choices of indices $\left\{j_{1}, j_{2}, \ldots, j_{q}\right\}$ among 1 and $k$ (notice that it vanishes for any $j_{i}=m$ ). We will demonstrate right ahead that the contributions to Eq. (8.3) due to $q=0,1, \ldots, k-2$ vanish and only the one due to $q=k-1$ survives, providing

$$
I_{k}=\sum_{m=1}^{k} \prod_{j \neq m} \frac{F_{j}-F_{m}}{F_{j}^{*}-F_{m}^{*}} \int d^{2} x e^{-i p x} \frac{x_{1}+i x_{2}}{x_{1}-i x_{2}}=\frac{4 \pi}{p^{2}} k\left(-e^{2 i \psi}\right)^{k} .
$$

The previous result can be reconstructed by recalling that $F_{j}$ is aligned with $l$ (see Eq. (2.8)). If we use a two-component vector notation and parametrize $\vec{p}=p(\cos \psi, \sin \psi)$, then $\vec{F}_{j}=$ $\left|F_{j}\right|(\sin \psi,-\cos \psi), \forall j$, so that Eq. (8.4) ensues. In order to prove Eq. (3.7), we remark that, in the case of an arbitrary angle $\psi$ between the lines and the reference frame, the factor $(-1)^{n-k}$ in Eq. (3.5), due to integrating over propagators starting and ending on the same line, is replaced by $\left(-e^{2 i \psi}\right)^{n-k}$. Eventually, when performing the integration over the $2 n$ line variables $\zeta^{+}, \zeta^{\prime+}$, Eq. (3.7) is recovered, since the factor $\left(-e^{2 i \psi}\right)^{n}$ cancels against its counterpart $\left(-e^{-2 i \psi}\right)^{n}$ originating from the $\zeta^{+}$integrations. Notice that Eq. (3.6) corresponds to the choice $\psi=0$.

To conclude our proof we have to show the following

$$
\sum_{m=1}^{k} \sum_{j_{1}<j_{2}<\ldots<j_{q}}\left(F_{j_{1}}-F_{m}\right)\left(F_{j_{2}}-F_{m}\right) \ldots\left(F_{j_{q}}-F_{m}\right) \prod_{j \neq m} \frac{1}{F_{j}^{*}-F_{m}^{*}}=0,
$$

for $q=0,1, \ldots, k-2$. We start by rewriting the l.h.s. of Eq. (8.5) as

$$
\begin{aligned}
& \left(-e^{2 i \psi}\right)^{q} \sum_{m=1}^{k} \sum_{i_{1}<i_{2}<\ldots<i_{k-q-1} \neq m} \frac{1}{F_{i_{1}}^{*}-F_{m}^{*}} \frac{1}{F_{i_{2}}^{*}-F_{m}^{*}} \cdots \frac{1}{F_{i_{n-q-1}}^{*}-F_{m}^{*}}= \\
& \left(-e^{2 i \psi}\right)^{q} \sum_{m=1}^{k} \sum_{i_{1}<i_{2}<\ldots<i_{k-q-1} \neq m} \frac{\Delta_{k-q-1}\left(F_{i_{1}}^{*}, F_{i_{2}}^{*}, \ldots, F_{i_{k-q-1}}^{*}\right)}{\Delta_{k-q}\left(F_{i_{1}}^{*}, F_{i_{2}}^{*}, \ldots, F_{i_{k-q-1}}^{*}, F_{m}^{*}\right)},
\end{aligned}
$$

where $\Delta_{l}$ is the Vandermonde determinant of $l$ elements. The sum is over all indices in $\{1, \ldots, k\}$ left over by the set $\left\{j_{1}, \ldots, j_{q}, m\right\}$, which have been labelled $\left\{i_{1}, \ldots, i_{k-q-1}\right\}$. In Eq. (8.6) the index $m$ is not ordered with respect to the $\left\{i_{l}\right\}$ 's. By exploiting the symmetry properties of the Vandermonde determinant in the denominator and relabelling $m=i_{k-q}$, we get (up to an overall phase)

$$
\begin{aligned}
& \sum_{i_{1}<i_{2}<\ldots<i_{k-q}} \Delta_{k-q}^{-1}\left(F_{i_{1}}^{*}, F_{i_{2}}^{*}, \ldots, F_{i_{k-q-1}}^{*}, F_{i_{k-q}}^{*}\right) \times \\
& \sum_{r=1}^{k-q}(-1)^{k-q-r} \Delta_{k-q-1}\left(F_{i_{1}}^{*}, \ldots, F_{i_{r-1}}^{*}, F_{i_{r+1}}^{*}, \ldots, F_{i_{k-q-1}}^{*}, F_{i_{k-q}}^{*}\right),
\end{aligned}
$$


which vanishes thanks to a well known property of determinants.

We conclude that Eq. (3.6) indeed holds.

\section{References}

[1] L. Alvarez-Gaume and S. R. Wadia, "Gauge theory on a quantum phase space", Phys. Lett. B 501 (2001) 319 [hep-th/0006219].

[2] J. A. Harvey, "Topology of the gauge group in noncommutative gauge theory" [hep-th/0105242].

[3] N. Ishibashi, S. Iso, H. Kawai and Y. Kitazawa, "Wilson loops in noncommutative Yang-Mills", Nucl. Phys. B 573 (2000) 573 [hep-th/9910004].

[4] S. J. Rey and R. von Unge, "S-duality, noncritical open string and noncommutative gauge theory", Phys. Lett. B 499 (2001) 215 [hep-th/0007089]; S. R. Das and S. J. Rey, "Open Wilson lines in noncommutative gauge theory and tomography of holographic dual supergravity", Nucl. Phys. B 590 (2000) 453 [hep-th/0008042].

[5] D. J. Gross, A. Hashimoto and N. Itzhaki, "Observables of non-commutative gauge theories", Adv. Theor. Math. Phys. 4 (2000) 893 [hep-th/0008075].

[6] G. 't Hooft, "A Two-Dimensional Model For Mesons", Nucl. Phys. B 75 (1974) 461.

[7] A. Schwarz, "Morita equivalence and duality", Nucl. Phys. B 534 (1998) 720 [hep-th/9805034]; B. Pioline and A. Schwarz, "Morita equivalence and T-duality (or $B$ versus $\Theta$ )", JHEP 9908 (1999) 021 [hep-th/9908019].

[8] A. A. Migdal, "Recursion Equations In Gauge Field Theories", Sov. Phys. JETP 42 (1975) 413 [Zh. Eksp. Teor. Fiz. 69 (1975) 810]; B. E. Rusakov, "Loop Averages And Partition Functions In U(N) Gauge Theory On Two-Dimensional Manifolds", Mod. Phys. Lett. A 5 (1990) 693.

[9] G. Mandal, S. J. Rey and S. R. Wadia, "Quantum aspects of GMS solutions of noncommutative field theory and large N limit of matrix models", Eur. Phys. J. C 24 (2002) 495 [hep-th/0111059].

[10] R. Gopakumar, S. Minwalla and A. Strominger, "Noncommutative solitons", JHEP 0005 (2000) 020 [hep-th/0003160].

[11] T. T. Wu, "Two-Dimensional Yang-Mills Theory In The Leading 1/N Expansion", Phys. Lett. B 71 (1977) 142.

[12] S. Mandelstam, "Light Cone Superspace And The Ultraviolet Finiteness Of The N=4 Model", Nucl. Phys. B 213 (1983) 149; G. Leibbrandt, "The Light Cone Gauge In Yang-Mills Theory", Phys. Rev. D 29 (1984) 1699. 
[13] A. Bassetto, G. Nardelli and R. Soldati, Yang-Mills Theories In Algebraic Noncovariant Gauges: Canonical Quantization And Renormalization, Singapore, World Scientific (1991) $227 \mathrm{p}$.

[14] A. Bassetto, M. Dalbosco, I. Lazzizzera and R. Soldati, "Yang-Mills Theories In The Light Cone Gauge", Phys. Rev. D 31 (1985) 2012.

[15] A. Bassetto, G. Nardelli and A. Torrielli, "Perturbative Wilson loop in two-dimensional non-commutative Yang-Mills theory," Nucl. Phys. B 617 (2001) 308 [hep-th/0107147].

[16] A. K. Das and M. M. Sheikh-Jabbari, "Absence of higher order corrections to noncommutative Chern-Simons coupling", JHEP 0106 (2001) 028 [hep-th/0103139].

[17] M. Staudacher and W. Krauth, "Two-Dimensional QCD in the Wu-Mandelstam-Leibbrandt Prescription", Phys. Rev. D57 (1998) 2456.

[18] K. Zarembo, "Supersymmetric Wilson loops" [hep-th/0205160].

[19] G. 't Hooft, "A Property Of Electric And Magnetic Flux In Nonabelian Gauge Theories", Nucl. Phys. B 153 (1979) 141.

[20] Z. Guralnik and J. Troost, "Aspects of gauge theory on commutative and noncommutative tori", JHEP 0105 (2001) 022 [hep-th/0103168]; Z. Guralnik, "Strong coupling phenomena on the noncommutative plane", JHEP 0206 (2002) 010 [hep-th/0109079].

[21] L. Griguolo, D. Seminara and P. Valtancoli, "Towards the solution of noncommutative $Y M_{2}$ : Morita equivalence and large N-limit", JHEP 0112 (2001) 024 [hep-th/0110293].

[22] J. Ambjorn, Y. M. Makeenko, J. Nishimura and R. J. Szabo, "Lattice gauge fields and discrete noncommutative Yang-Mills theory", JHEP 0005 (2000) 023 [hep-th/0004147]; K. Saraikin, "Comments on the Morita equivalence", J. Exp. Theor. Phys. 91 (2000) 653 [Zh. Eksp. Teor. Fiz. 91 (2000) 755] [hep-th/0005138]. 